\title{
Ag Concentration Dependence of Build-up Effect of Radio-photoluminescence in Ag-doped $\mathrm{P}_{2} \mathrm{O}_{5}-\mathrm{Al}_{2} \mathrm{O}_{3}-\mathrm{Na}_{2} \mathrm{O}-\mathrm{SiO}_{2}$ Glasses
}

\author{
Takumi Kato, ${ }^{1 *}$ Daiki Shiratori, ${ }^{1}$ Masaru Iwao, ${ }^{2}$ Hironori Takase, ${ }^{2}$ \\ Daisuke Nakauchi, ${ }^{1}$ Noriaki Kawaguchi, ${ }^{1}$ and Takayuki Yanagida ${ }^{1}$ \\ ${ }^{1}$ Nara Institute of Science and Technology, 8916-5 Takayama-cho, Ikoma-shi, Nara 630-0192, Japan \\ ${ }^{2}$ Nippon Electric Glass Co., Ltd., 7-1, Seiran 2-chome, Otsu-shi, Shiga 520-8639, Japan
}

(Received January 28, 2021; accepted May 10, 2021)

Keywords: build-up, radio-photoluminescence, silver, glass

In this paper, we evaluated the Ag concentration dependence of the build-up effect of radiophotoluminescence (RPL) in Ag-doped $\mathrm{P}_{2} \mathrm{O}_{5}-\mathrm{Al}_{2} \mathrm{O}_{3}-\mathrm{Na}_{2} \mathrm{O}-\mathrm{SiO}_{2}$ (PANS) glasses. After X-ray irradiation, the Ag-doped PANS glasses showed two emission peaks at around 460 and $630 \mathrm{~nm}$, assigned to blue and orange RPL components, respectively. The build-up curves of the orange RPL component had two components corresponding to the formation of the $\mathrm{Ag}_{2}^{+}$dimer and $\mathrm{Ag}^{2+}$ ions. The intensity of the component due to the $\mathrm{Ag}_{2}^{+}$dimer increased with increasing $\mathrm{Ag}$ concentration, whereas that of the component due to $\mathrm{Ag}^{2+}$ ions decreased. It is assumed that at a high $\mathrm{Ag}$ concentration, the $\mathrm{Ag}^{+}$ions have a higher probability of diffusing to $\mathrm{Ag}^{0}$ sites to form the $\mathrm{Ag}_{2}^{+}$dimer because the average distance between neighboring $\mathrm{Ag}$ atoms in the glass structure decreases with increasing Ag concentration. Therefore, the intensity of the component due to the $\mathrm{Ag}_{2}^{+}$dimer was dominant at a high concentration of $\mathrm{Ag}$ in the glass structure.

\section{Introduction}

Radio-photoluminescence (RPL) is a luminescence phenomenon in which photoluminescence (PL) centers are newly generated by the irradiation of phosphors. ${ }^{(1-3)}$ Since the PL intensity is proportional to the absorbed radiation dose, RPL can be applied to dosimetry. PL centers in RPL do not disappear after the dose information is read out because the readout obtained with RPL does not depend on the recombination of electrons and holes stored at trapping centers, unlike in the case of thermally stimulated luminescence (TSL) and optically stimulated luminescence (OSL). Thus, the RPL intensity can be repeatably read out, making it advantageous over TSL and OSL.(4) Phosphors exhibiting RPL include Ag-doped phosphate glasses, ${ }^{(5)} \mathrm{Cu}$-doped glass, ${ }^{(6,7)} \mathrm{Al}_{2} \mathrm{O}_{3}: \mathrm{C}, \mathrm{Mg},{ }^{(8)}$ Eu-doped materials, ${ }^{(9,10)} \mathrm{Sm}$-doped materials, ${ }^{(11,12)} \mathrm{Yb}$-doped $\mathrm{NaCl}^{(13)}$ and $\mathrm{LiF}_{,}{ }^{(14,15)} \mathrm{MgF}_{2},{ }^{(16)} \mathrm{CaF}_{2},{ }^{(17,18)} \mathrm{Mg}_{2} \mathrm{SiO}_{4},{ }^{(19)} \mathrm{CaSO}_{4},{ }^{(20)} \mathrm{K}_{2} \mathrm{CO}_{3},{ }^{(21)}$ and $\mathrm{Na}_{2} \mathrm{CO}_{3} .{ }^{(22)}$

The phosphors exhibiting RPL of greatest interest are currently Ag-doped phosphate glasses. Since the first report on RPL by Schulman et al. in 1951, ${ }^{(23)}$ Ag-doped phosphate glasses have been studied and were commercialized by Chiyoda Technol Corporation as an RPL dosimeter 
called Glass Badge. The Ag doped in the phosphate glass exists stably and uniformly in the form of $\mathrm{Ag}^{+}$ions. After irradiation, $\mathrm{Ag}^{+}$ions in the phosphate glass capture electrons or holes generated by the radiation, and part of the $\mathrm{Ag}^{+}$ions change to $\mathrm{Ag}^{0}$ by capturing an electron $\left(\mathrm{Ag}^{+}+\mathrm{e}^{-} \rightarrow \mathrm{Ag}^{0}\right)$. On the other hand, part of the generated holes are captured at $\mathrm{PO}_{4}^{3-}$ tetrahedra and are transferred to $\mathrm{Ag}^{+}$ions upon thermal treatment, and part of the $\mathrm{Ag}^{+}$ions become $\mathrm{Ag}^{2+}$ ions by capturing a hole $\left(\mathrm{Ag}^{+}+\mathrm{h} \rightarrow \mathrm{Ag}^{2+}\right)$. The RPL intensity continues to increase following the cessation of irradiation owing to the transfer of holes, and the increase saturates after some hours at room temperature. This well-known phenomenon is termed the build-up effect.

The chemical composition of Ag-doped phosphate glass has become standardized since Glass Badge was widely applied for personal dosimetry because of its high reliability. However, abnormal luminescent signals caused by the degraded surface of the glass have been reported. ${ }^{(24-26)}$ This phenomenon is considered to occur when the surface degradation of the glass is accelerated by a humid environment. From the perspective of glass structure science, the network structure of the phosphate glass has poor weathering resistance because the $\mathrm{P}-\mathrm{O}-\mathrm{P}$ bond (phosphate-oxygen bond) is disconnected by the attack of $\mathrm{OH}^{-}$ions in the atmosphere. ${ }^{(27)}$ To achieve a strong network structure of the phosphate glass, $\mathrm{Al}_{2} \mathrm{O}_{3}$ and $\mathrm{SiO}_{2}$ are generally added. ${ }^{(28,29)}$

Therefore, we have developed Ag-doped phosphate glasses containing $\mathrm{SiO}_{2}$ and more $\mathrm{Al}_{2} \mathrm{O}_{3}$ than those in the conventional RPL dosimeter. ${ }^{(30)}$ As a result of examining their RPL properties, we found that the RPL properties of the developed glasses were equivalent to those of the existing RPL dosimeter and had superior weathering resistance to the conventional phosphate glass. In this study, we evaluated the Ag concentration dependence of the build-up effect in $\mathrm{P}_{2} \mathrm{O}_{5}-\mathrm{Al}_{2} \mathrm{O}_{3}-\mathrm{Na}_{2} \mathrm{O}-\mathrm{SiO}_{2}$ (PANS) glasses. It has already been reported that the main factors affecting the rate of build-up are the temperature, chemical composition of the glass, and $\mathrm{Ag}$ concentration. ${ }^{(31-36)}$ However, the reason why the rate of build-up depends on the Ag concentration has not yet been investigated.

\section{Materials and Methods}

A commercially available GD-450 dosimeter (AGC Techno Glass Co. Ltd.) was used as the reference sample of the conventional Ag-doped phosphate glass. This sample has a composition of $59 \mathrm{P}_{2} \mathrm{O}_{5}-13.2 \mathrm{Al}_{2} \mathrm{O}_{3}-27.7 \mathrm{Na}_{2} \mathrm{O}-0.1 \mathrm{Ag}_{2} \mathrm{O}$. In this work, PANS glasses with different concentrations $(0.05,0.1,0.2$, and $0.4 \mathrm{~mol} \%)$ of $\mathrm{Ag}_{2} \mathrm{O}$ as an activator were prepared from the starting materials of $\mathrm{Na}_{3} \mathrm{PO}_{4}$ (98.6\%), $\mathrm{AlPO}_{4}$ (99.4\%), $\mathrm{SiO}_{2}$ (99.9\%), and $\mathrm{Ag}_{2} \mathrm{O}$ (99.9\%). The concentrations of $\mathrm{SiO}_{2}$ and $\mathrm{Al}_{2} \mathrm{O}_{3}$ in the PANS glasses were set to 5 and $19 \mathrm{~mol} \%$, respectively.

The Ag-doped PANS glass samples were synthesized by the following method. Raw powders of $\mathrm{Na}_{3} \mathrm{PO}_{4}, \mathrm{AlPO}_{4}, \mathrm{SiO}_{2}$, and $\mathrm{Ag}_{2} \mathrm{O}$ were homogeneously mixed and melted at $1200{ }^{\circ} \mathrm{C}$ in an electric furnace for $3 \mathrm{~h}$. The melted mixture was cooled rapidly in a carbon mold, and the obtained glass ingots were annealed at approximately $500{ }^{\circ} \mathrm{C}$ for $1 \mathrm{~h}$ in a furnace. After annealing, the glass samples were cut into $30 \times 7 \times 1 \mathrm{~mm}^{3}$ pieces and polished using grinding equipment. All glass samples were washed and cleaned using deionized water and ethanol solution before every evaluation. 
To observe the build-up effect of RPL in each sample, PL emission spectra were obtained by a spectrofluorometer (FP8600, JASCO) at room temperature after X-ray irradiation. The excitation wavelength was fixed to $310 \mathrm{~nm}$, and the emission spectra were obtained in the wavelength range from 400 to $800 \mathrm{~nm}$ until $14 \mathrm{~h}$ after X-ray irradiation. To separate the excitation light and the PL, a band-pass filter (BPF340, ASAHI SPECTRA) and short-cut filter (SCF400, ASAHI SPECTRA) were also used to obtain the PL emission spectrum. An X-ray generator (XRB80N100/CB, Spellman), which was equipped with an X-ray tube having a W anode target and a Be window, was used as the radiation source. The X-ray tube was operated with a bias voltage of $40 \mathrm{kV}$ and a tube current of $5.2 \mathrm{~mA}$. The irradiation dose was calibrated using an air-filled ionization chamber (TN30013, PTW).

\section{Results and Discussion}

Figure 1 shows the PL emission spectra after X-ray irradiation of the (a) 0.05, (b) 0.1, (c) 0.2, and (d) $0.4 \mathrm{~mol} \% \mathrm{Ag}$-doped PANS samples and (e) Glass Badge. The irradiation dose was $10 \mathrm{~Gy}$ in all the measurements. In each figure, " 0 h" indicates the spectrum measured soon after X-ray irradiation. The spectral features of the Ag-doped PANS samples were consistent with those of the reference sample (Glass Badge), and two emission peaks were detected at around 460 and $630 \mathrm{~nm}$ in all the samples. These are assigned to blue and orange RPL components, which are due to $\mathrm{Ag}^{0}$ and mainly $\mathrm{Ag}^{2+}$ ions, respectively. ${ }^{(3,37,38)}$ In addition, it has recently been suggested that the orange RPL component also includes an emission peak due to the $\mathrm{Ag}_{2}^{+}$dimer. ${ }^{(5,31,39)}$

Figure 2 shows the build-up curves of RPL for each sample. In this figure, the horizontal axis indicates integrated values in the PL emission spectra (Fig. 1) from 500 to $800 \mathrm{~nm}$. An increase in the PL intensity of the orange RPL component with increasing elapsed time was clearly

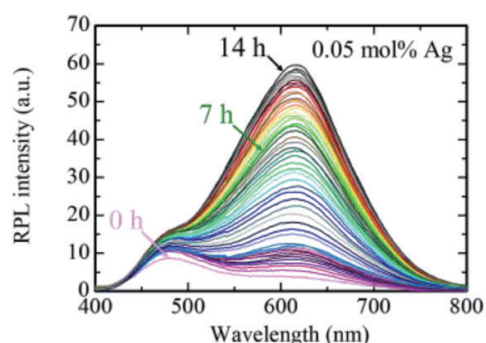

(a)

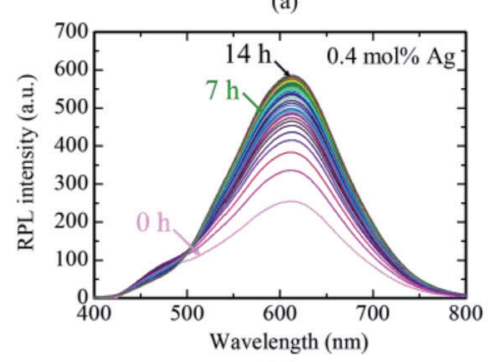

(d)

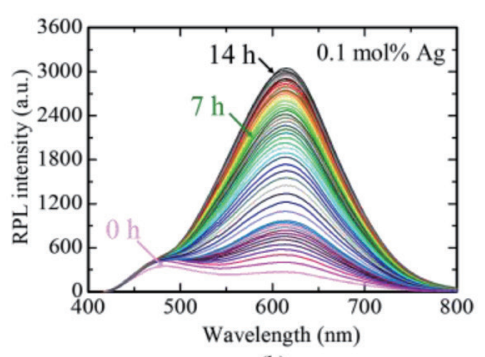

(b)

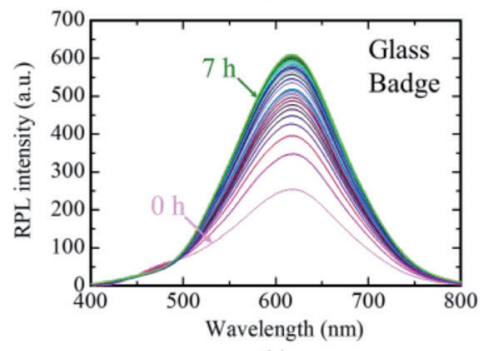

(e)

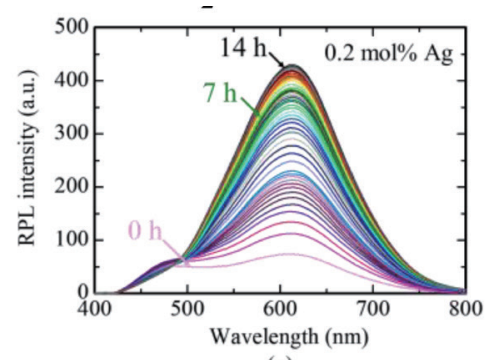

(c)

Fig. 1. (Color online) PL emission spectra after X-ray irradiation of the (a) 0.05, (b) 0.1, (c) 0.2, and (d) $0.4 \mathrm{~mol} \%$ Ag-doped PANS samples and (e) Glass Badge. 


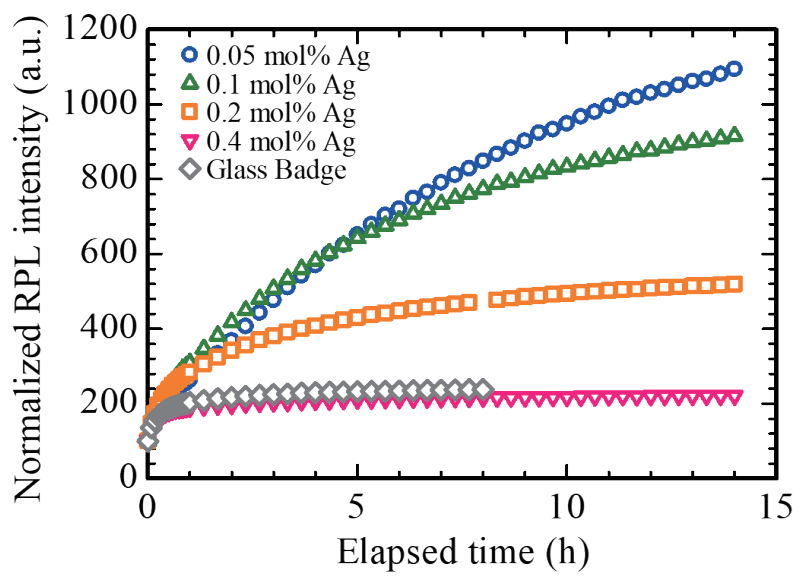

Fig. 2. (Color online) Build-up curves of RPL of all the samples.

observed for all the samples. The RPL intensity of the $0.05 \mathrm{~mol} \%$ Ag-doped PANS sample increased immediately after the irradiation and continued to increase even after $14 \mathrm{~h}$, whereas that of the $0.4 \mathrm{~mol} \%$ Ag-doped PANS sample showed a constant value after $1-2 \mathrm{~h}$. As a result, the time required for the build-up to saturate decreased with increasing Ag concentration. In addition, the build-up curve of the PANS sample doped with the largest Ag concentration (0.4 mol\%) showed similar behavior to that of Glass Badge.

To analyze the experimentally obtained build-up curves in more detail, we performed a fitting analysis. However, as pointed out in a previous report, ${ }^{(31)}$ it is difficult to obtain an analytical equation to describe the shape of these curves without a clear physical model to explain the build-up process. Thus, we performed the fitting analysis using the empirical equation

$$
I_{\mathrm{BU}}(t)=\sum_{i=1}^{n}\left[-A_{i} \exp \left(-t / \tau_{i}\right)+A_{i}\right]+\text { Const. }
$$

where $I_{\mathrm{BU}}(t)$ is the intensity of the RPL during build-up, $t$ is the elapsed time after irradiation, and $A_{i}$ and $\tau_{i}$ are the intensity and build-up time constants for each component, respectively.

Figure 3 shows the build-up curves of each sample with the fitting functions. The build-up curve of the $0.05 \mathrm{~mol} \% \mathrm{Ag}$-doped sample was best fitted by a single component, whereas those of the other samples were best fitted by the sum of two components. The fitting parameters are summarized in Table 1. According to a recent study, ${ }^{(31)}$ the build-up curve of Ag-doped phosphate glass includes two components corresponding to the formation of the $\mathrm{Ag}_{2}^{+}$dimer and $\mathrm{Ag}^{2+}$ ions. The fitting results for the 0.1, 0.2, and $0.4 \mathrm{~mol} \%$ Ag-doped PANS samples and Glass Badge in this study are consistent with those in Ref. 31, in which it was clarified that the first (fast) and second (slow) components are caused by the $\mathrm{Ag}_{2}^{+}$dimer and $\mathrm{Ag}^{2+}$ ions, respectively. Thus, the first (slow) component of the $0.05 \mathrm{~mol} \%$ Ag-doped PANS sample was considered to be due to $\mathrm{Ag}^{2+}$ ions. 


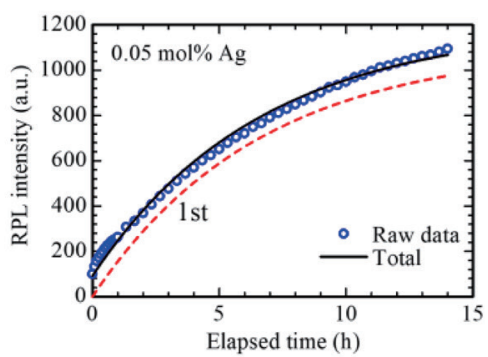

(a)

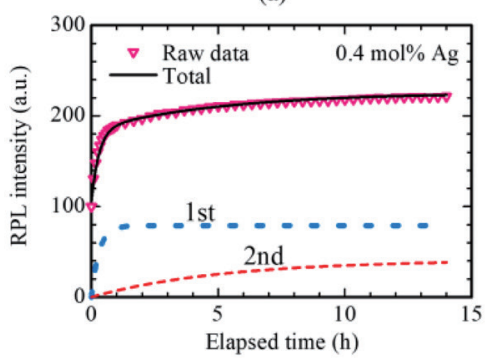

(d)

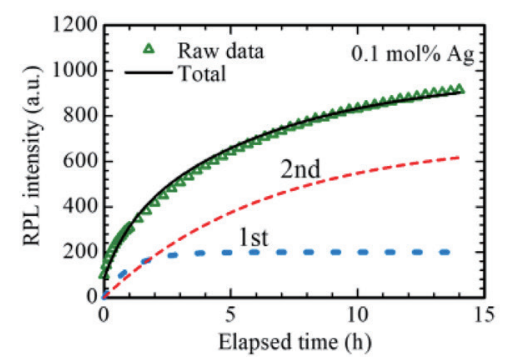

(b)

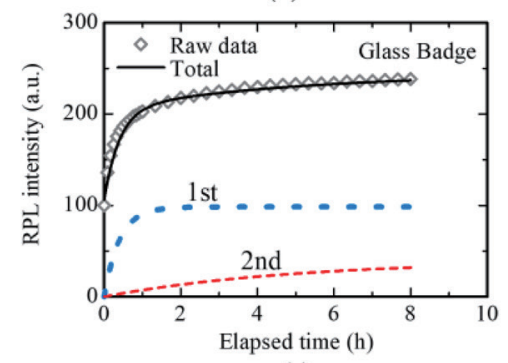

(e)

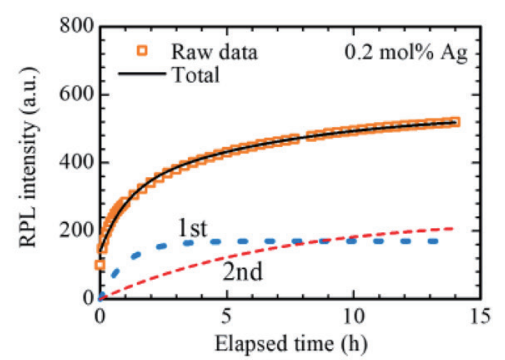

(c)

Fig. 3. (Color online) Build-up curves with fitting functions of each sample: (a) 0.05, (b) 0.1, (c) 0.2, and (d) 0.4 mol\% Ag-doped PANS samples and (e) Glass Badge.

Table 1

Fitting parameters of each build-up curve.

\begin{tabular}{lccrr}
\hline & $\tau_{1}(\mathrm{~h})$ & $\tau_{2}(\mathrm{~h})$ & \multicolumn{1}{c}{$A_{1}$} & \multicolumn{1}{c}{$A_{2}$} \\
\hline $0.05 \mathrm{~mol} \% \mathrm{Ag}$ & 6.6 & - & 1108.4 & - \\
$0.1 \mathrm{~mol} \% \mathrm{Ag}$ & 1.0 & 6.5 & 199.9 & 697.9 \\
$0.2 \mathrm{~mol} \% \mathrm{Ag}$ & 1.0 & 7.0 & 170.0 & 239.8 \\
$0.4 \mathrm{~mol} \% \mathrm{Ag}$ & 0.3 & 5.2 & 79.0 & 41.0 \\
Glass Badge & 0.4 & 5.0 & 98.5 & 40.0 \\
\hline
\end{tabular}

A clear difference was observed between the build-up curves of the $0.1 \mathrm{~mol} \%$ Ag-doped sample and Glass Badge, even though the $\mathrm{Ag}$ concentrations of the two samples were the same. This is because the presence of $\mathrm{Si}$ and $\mathrm{Al}$ in the phosphate glass not only strengthened the network of the phosphate glass but also increased the activation energy for the diffusion of $\mathrm{Ag}^{+}$ ions. ${ }^{(30)}$ Another possibility is that the activation energy for the thermal release of holes from $\mathrm{PO}_{4}^{2-}$ in the glass structure changes with the introduction of $\mathrm{Si}$ and $\mathrm{Al}$.

Figure 4 presents ratios of the intensities of the fast and slow components as a function of the $\mathrm{Ag}$ concentration. For the $0.05 \mathrm{~mol} \%$ Ag-doped PANS sample, the ratio is $100 \%$ for the slow component because only the slow component was obtained. The intensity of the fast component due to the $\mathrm{Ag}_{2}^{+}$dimer increased with increasing $\mathrm{Ag}$ concentration. The average distance between neighboring Ag atoms, in the glass structure decreases with increasing Ag concentration, increasing the probability of the $\mathrm{Ag}_{2}^{+}$dimer being formed. ${ }^{(35)}$ Hence, the intensity of the fast component due to the $\mathrm{Ag}_{2}^{+}$dimer was dominant for a high $\mathrm{Ag}$ concentration in the glass structure. It was previously reported that the $\mathrm{Ag}$ concentration dependence of the build-up effect is only associated with the amount of $\mathrm{Ag}^{2+}$ ions generated. ${ }^{(36)}$ However, the present results 


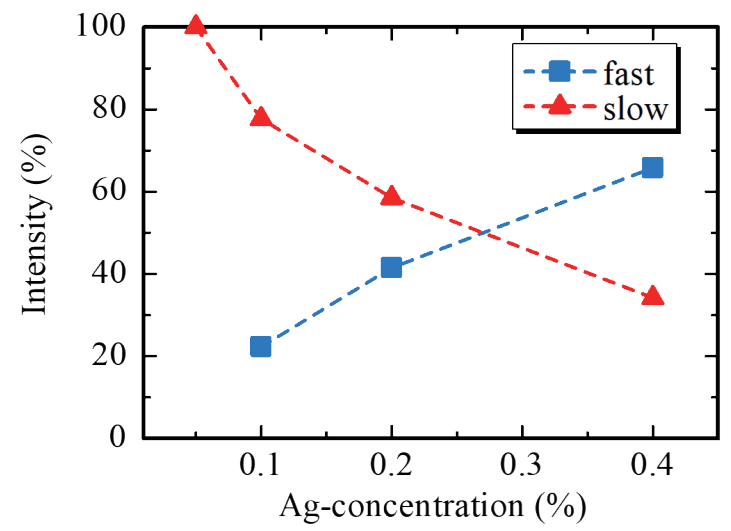

Fig. 4. (Color online) Ratios of the intensities of the fast and slow components as a function of the Ag concentration.

suggest that the $\mathrm{Ag}$ concentration dependence of the build-up effect is related to not only $\mathrm{Ag}^{2+}$ ions but also the $\mathrm{Ag}_{2}^{+}$dimer.

\section{Conclusions}

We investigated the Ag concentration dependence of the build-up effect in Ag-doped $\mathrm{P}_{2} \mathrm{O}_{5}-$ $\mathrm{Al}_{2} \mathrm{O}_{3}-\mathrm{Na}_{2} \mathrm{O}-\mathrm{SiO}_{2}$ glasses. The build-up curves of the orange RPL component included fast and slow components, which were attributable to the formation of the $\mathrm{Ag}_{2}^{+}$dimer and $\mathrm{Ag}^{2+}$ ions, respectively. The intensity of the component due to the $\mathrm{Ag}_{2}^{+}$dimer increased with increasing $\mathrm{Ag}$ concentration. In other words, the component due to the $\mathrm{Ag}_{2}^{+}$dimer becomes dominant when the glass contains more $\mathrm{Ag}^{+}$ions. Because the component due to the $\mathrm{Ag}_{2}^{+}$dimer was faster than the component due to $\mathrm{Ag}^{2+}$ ions, the present results show that increasing the $\mathrm{Ag}^{+}$ion concentration in the glass structure could shorten the time before build-up saturates.

\section{Acknowledgments}

This work was supported by Grants-in-Aid for Scientific Research A (17H01375) and B (18H03468 and 19H03533) and Early-Career Scientists (20K15026) from JSPS. The Cooperative Research Project of the Research Institute of Electronics, Shizuoka University, and Murata Foundation are also acknowledged.

\section{References}

1 Y. Miyamoto, T. Yamamoto, K. Kinoshita, S. Koyama, Y. Takei, H. Nanto, Y. Shimotsuma, M. Sakakura, K. Miura, and K. Hirao: Radiat. Meas. 45 (2010) 546.

2 Y. Miyamoto, Y. Takei, H. Nanto, T. Kurobori, A. Konnai, T. Yanagida, A. Yoshikawa, Y. Shimotsuma, M. Sakakura, K. Miura, K. Hirao, Y. Nagashima, and T. Yamamoto: Radiat. Meas. 46 (2011) 1480.

3 T. Yamamoto, D. Maki, F. Sato, Y. Miyamoto, H. Nanto, and T. Iida: Radiat. Meas. 46 (2011) 1554.

4 T. Yanagida, G. Okada, and N. Kawaguchi: J. Lumin. 207 (2019) 14.

5 T. Kurobori, W. Zheng, Y. Miyamoto, H. Nanto, and T. Yamamoto: Opt. Mater. (Amst). 32 (2010) 1231. 
6 R. Hashikawa, Y. Fujii, A. Kinomura, T. Saito, A. Okada, T. Wakasugi, and K. Kadono: J. Am. Ceram. Soc. $102(2019) 1642$.

7 H. Masai, H. Ofuchi, G. Okada, N. Kawaguchi, and T. Yanagida: Sens. Mater. 31 (2019) 1297.

8 M. S. Akselrod and A. E. Akselrod: Radiat. Prot. Dosimetry 119 (2006) 218.

9 S. Asada, G. Okada, T. Kato, F. Nakamura, N. Kawano, N. Kawaguchi, and T. Yanagida: Chem. Lett. 47 (2018) 59.

10 R. L. Calvert and R. J. Danby: Phys. Status Solidi 83 (1984) 597.

11 G. Okada, Y. Fujimoto, H. Tanaka, S. Kasap, and T. Yanagida: J. Rare Earths 34 (2016) 769.

12 G. Okada, K. Shinozaki, T. Komatsu, S. Kasap, and T. Yanagida: Radiat. Meas. 106 (2017) 73.

13 Y. Fujimoto, G. Okada, D. Sekine, T. Yanagida, M. Koshimizu, H. Kawamoto, and K. Asai: Radiat. Meas. 133 (2020) 106274.

14 Z. Knežević, N. Beck, D. Milković, S. Miljanić, and M. Ranogajec-Komor: Radiat. Meas. 46 (2011) 1582.

15 M. Levita, T. Schlesinger, and S. S. Friedland: IEEE Trans. Nucl. Sci. 23 (1976) 667.

16 F. Nakamura, T. Kato, G. Okada, N. Kawaguchi, K. Fukuda, and T. Yanagida: Ceram. Int. 43 (2017) 7211.

17 T. Kato, D. Nakauchi, N. Kawaguchi, and T. Yanagida: Mater. Lett. 270 (2020) 127688.

18 T. Kato, D. Nakauchi, N. Kawaguchi, and T. Yanagida: Curr. Appl. Phys. 20 (2020) 1195.

19 G. Okada, T. Kojima, J. Ushizawa, N. Kawaguchi, and T. Yanagida: Curr. Appl. Phys. 17 (2017) 422.

20 G. Okada, Y. Koguchi, T. Yanagida, and H. Nanto: Mater. Today Commun. 24 (2020) 101013.

21 F. Nakamura, T. Kato, G. Okada, N. Kawano, N. Kawaguchi, and T. Yanagida: Mater. Lett. 211 (2018) 100.

22 F. Nakamura, T. Kato, D. Nakauchi, G. Okada, N. Kawano, N. Kawaguchi, and T. Yanagida: Chem. Lett. 46 (2017) 1383.

23 J. H. Schulman, R. J. Ginther, C. C. Klick, R. S. Alger, and R. A. Levy: J. Appl. Phys. 22 (1951) 1479.

24 H. Yamanishi, H. TadashiMiyake, T. Yamasaki, and K. Komura: Hoken Butsuri 38 (2003) 45 (in Japanese).

25 S. Hashimoto, T. Hashimoto, M. Ishikawa, and M. Emori: Hoken Butsuri 39 (2004) 231 (in Japanese).

26 R. Matthews, D. R. Weavert, and S. Croft: Radiat. Prot. Dosimetry 65 (1996) 301.

27 B. C. Bunker, G. W. Arnold, and J. A. Wilder: J. Non. Cryst. Solids 64 (1984) 291.

28 A. A. El-Kheshen, F. A. Khaliafa, E. A. Saad, and R. L. Elwan: Ceram. Int. 34 (2008) 1667.

29 F. Wu, S. Li, Z. Chang, H. Liu, S. Huang, and Y. Yue: J. Mol. Struct. 1118 (2016) 42.

30 M. Iwao, H. Takase, D. Shiratori, D. Nakauchi, T. Kato, N. Kawaguchi, and T. Yanagida: Radiat. Meas. 140 (2021) 106492.

31 S. W. S. McKeever, S. Sholom, N. Shrestha, and D. M. Klein: Radiat. Meas. 132 (2020) 106246.

32 T. Kurobori: Jpn. J. Appl. Phys. 57 (2018) 106402.

33 F. Sato, N. Zushi, T. Nagai, T. Tanaka, Y. Kato, T. Yamamoto, and T. Iida: Radiat. Meas. 53-54 (2013) 8.

34 A. V. Dmitryuk, S. E. Paramzina, A. S. Perminov, N. D. Solov'eva, and N. T. Timofeev: J. Non. Cryst. Solids 202 (1996) 173.

35 S. M. Hsu, S. W. Yung, R. K. Brow, W. L. Hsu, C. C. Lu, F. B. Wu, and S. H. Ching: Mater. Chem. Phys. 123 (2010) 172.

36 R. Yokota and H. Imagawa: J. Phys. Soc. Jpn. 23 (1966) 1038.

37 Y. Miyamoto, T. Ohno, Y. Takei, H. Nanto, T. Kurobori, T. Yanagida, A. Yoshikawa, Y. Nagashima, and T. Yamamoto: Radiat. Meas. 55 (2013) 72.

38 T. Kato, D. Shiratori, D. Nakauchi, N. Kawaguchi, and T. Yanagida: Jpn. J. Appl. Phys. 59 (2020) 112001.

39 S. W. S. McKeever, S. Sholom, and N. Shrestha: Radiat. Meas. 123 (2019) 13. 